\title{
Clinical Criteria for the Diagnosis of Parkinson's Disease
}

\author{
Heinz Reichmann \\ Klinik und Poliklinik für Neurologie, Universitätsklinikum Carl Gustav Carus, Dresden, Germany
}

\section{Key Words}

Parkinson's disease $\cdot$ Hyposmia $\cdot$ REM sleep behavior

disorder $\cdot$ Diagnostic tests

\begin{abstract}
The diagnosis of Parkinson's disease (PD) follows the UK Brain Bank Criteria, which demands bradykinesia and one additional symptom, i.e. rigidity, resting tremor or postural instability. The latter is not a useful sign for the early diagnosis of PD, because it does not appear before Hoehn and Yahr stage 3. Early symptoms of PD which precede the onset of motor symptoms are hyposmia, REM sleep behavioral disorder, constipation, and depression. In addition, an increasing number of patients whose PD is related to a genetic defect are being described. Thus, genetic testing may eventually develop into a tool to identify at-risk patients. The clinical diagnosis of PD can be supported by levodopa or apomorphine tests. Imaging studies such as cranial CT or MRI are helpful to distinguish idiopathic PD from atypical or secondary PD. SPECT and PET methods are valuable to distinguish PD tremor from essential tremor if this is clinically not possible. Using all of these methods, we may soon be able to make a premotor diagnosis of $\mathrm{PD}$, which will raise the question whether early treatment is possible and ethically and clinically advisable.

Copyright $\odot 2010$ S. Karger AG, Basel
\end{abstract}

\section{Classical Clinical Diagnosis of Parkinson's Disease}

Parkinson's disease (PD) is considered to be the second most common neurodegenerative disease with an onset in the 5th or 6th decade. It is estimated to affect about $1-2 \%$ of individuals in this age group [1]. According to the UK PD Brain Bank criteria, the diagnosis of PD is based on three steps. First, the diagnosis of PD can be made if bradykinesia is associated with another main symptom, i.e. rigidity, tremor or postural instability [2]. Second, one has to check the exclusion criteria. Typical examples are a history of repeated strokes with stepwise progression of parkinsonian features, multiple head injuries, encephalitis, oculogyric crisis, neuroleptic treatment, toxins like manganese, carbon monoxide or MPTP, cerebral tumor, communicating hydrocephalus on CT scans, strictly unilateral features after 3 years, sustained remission, more than one relative affected with PD, and typical features of atypical PD such as cerebellar, pyramidal or oculomotor symptoms. The third step involves the ascertainment of supportive criteria for PD. Among these, unilateral onset and persistent asymmetry, excellent response to levodopa, severe levodopa-induced dyskinesia, and disease progression support a diagnosis of PD. These criteria are used worldwide both for clinical diagnosis in private practice and hospitals and also for clinical trials. Despite

\section{KARGER}

Fax +4161306 1234 E-Mail karger@karger.ch www.karger.com
Prof. Dr. med. Heinz Reichmann, Klinik und Poliklinik für Neurologie Universitätsklinikum Carl Gustav Carus, Technische Universität Dresden Fetscherstrasse 74, DE-01307 Dresden (Germany)

Tel. +49351 458 3565, Fax +49351 4584365

E-Mail heinz.reichmann@mailbox.tu-dresden.de 
the existence of these carefully established criteria, the authors themselves [2] emphasize that the correlation between clinical signs and neuropathology with Lewy bodies is not very strong. If clinical examination is the only diagnostic tool applied, Lewy bodies are only found in $76 \%$ of patients who were diagnosed with PD [3]. It should be noted, however, that the UK PD Brain Bank Criteria were developed in a restrictive context for research purposes.

Despite the widespread use of these criteria, there are also certain drawbacks. Postural instability, for example, is not a very useful clinical symptom at the onset of the disease when the diagnosis still has to be established. This symptom does not manifest before the patient has reached Hoehn and Yahr (HY) stage 3, which is an advanced stage [4]. Therefore, postural instability is not a good tool for early diagnosis. Modern treatment may even postpone the onset of HY stage 3, and therefore other symptoms may turn out to be more helpful for diagnosis.

The initial clinical diagnosis of PD is based on the presence of resting tremor, micrographia, hypomimia, impaired arm swing, muscle pain, and slowed repetitive movements.

\section{Tremor}

In most cases, resting tremor starts in the upper extremities and is unilateral (HY stage 1). Thereafter, it spreads to the other upper limb or to the ipsilateral lower limb. There are, however, also patients with initial tremor in the lower limbs, and it is quite possible that we often miss this site because of the larger weight of the legs. At the beginning, tremor only occurs intermittently [5], which explains why some patients notice their tremor first at times of stress. There are reports that over the years tremor may even decline when the patients enter the late stages of PD [6]. Typically, PD is associated with resting tremor, but action tremor is also found in many patients [7]. A good clinical test to unmask existing resting tremor is serial subtraction (e.g. of 7 from 100) with the eyes closed. The relationship between essential tremor (ET) with action tremor and PD with resting tremor is not a simple one because there are many patients with 'mixed' tremor who may develop an apparently more benign type of PD [8]. PET studies indicate that tremor is not as closely related to the decrease in dopamine production as are bradykinesia and rigidity [9], which is also suggested by the decline in tremor in late stages. One should be cautious, however, of the fact that some patients with ET may later also develop resting tremor [10], which does not mean that they have developed PD.

Diagnosis of Parkinson’s Disease

\section{Bradykinesia}

The term akinesia should be avoided, because most patients are not really akinetic, at least not in the first years of their PD career. Thus, bradykinesia may be the better alternative. Some authors, however, use the term akinesia to describe the inability to initiate movements and not as a description of total immobility. Marsden [11] defined bradykinesia as slowness of speed, which describes the most common clinical symptom in PD best. Therefore, the UK PD Brain Bank Criteria define bradykinesia as the most important feature for the diagnosis of PD [2]. Clinical expressions of bradykinesia are hypomimia, which is commonly more prominent on one side of the face, reduced arm swing on one side, which is often misinterpreted by patients and physicians as an orthopedic problem, or problems with writing and cutting food, to name just a few. In particular, repetitive movements are impaired, and in my own practice I consider reduced arm swing and impaired repetitive movements of the hands (pronation/supination) as one of the most useful features both for the diagnosis of PD and for the assessment of the effects of treatment. Handwriting should always be tested by asking the patient to write a given sentence five times, because the amplitude declines with the number of repeats. As mentioned above, the severity of bradykinesia correlates very well with the dopaminergic deficit $[9,12]$.

\section{Rigidity}

Testing for rigidity should be done with irregular movements to avoid counteraction or facilitation by the patient. Rigidity is noticed as a permanently increased muscle tone independent of stretching the agonist or antagonist muscle. Cogwheel rigidity is normally encountered in more advanced stages, but is very characteristic of PD. In particular, asymmetric rigidity is a strong indicator of PD. In case of uncertainty about the presence of rigidity, the Froment maneuver should be applied; this consists in voluntary movement of one limb whilst the investigator checks for a resulting increase in muscle tone in the opposite limb [13]. Confusion and uncertainty develops when patients with ET also present with a cogwheeling phenomenon, which is caused by intermittent increases of tremor when the examiner performs repetitive passive movements.

\section{Postural Instability}

As mentioned above, postural instability is not a very useful cardinal symptom in the early stages of PD, because, by definition, this symptom does not occur before 
HY stage 3 . With the pull test, the examiner should inform the patient to resist a sudden pull or make a correcting step if necessary. Taking more than one and especially more than four steps to prevent falling is considered as impaired postural control [14]. Postural instability presents on average 5 years after the onset of PD [15]. Early presentation with postural instability and gait disorders may be indicative of progressive supranuclear palsy $[16,17]$. It is important to remember that up to $70 \%$ of the elderly have impaired postural reflexes, which is another reason why in our view this so-called cardinal symptom is misleading [14]. On the contrary, it may be the cause for many false-positive diagnoses.

\section{Premotor Signs in PD}

The most recent studies have indicated that several non-motor symptoms are associated with an increased risk for the later development of PD. More than $90 \%$ of all $\mathrm{PD}$ patients present with non-motor symptoms during the course of their disease [18]. According to Braak et al. [19], PD affects not only the dopaminergic neurons, but also glutamatergic, cholinergic, serotonergic and adrenergic neurons in the brain and in the autonomic nervous system [20]. In the late 1980s, Wakabayashi et al. [21] already described abnormalities of the autonomic nervous system, which was corroborated by more recent studies by Braak et al. [22]. Table 1 gives a short summary of the most frequent non-motor features in PD. James Parkinson claimed in his Essay on the Shaking Palsy that the disease does not affect the senses and intellect; unfortunately this is not correct. It is our belief that because hyposmia is present in more than $90 \%$ of all PD patients, it should be regarded as a new cardinal symptom [23]. One has to consider, however, that only about $60 \%$ of our patients actually noticed their hyposmia or anosmia [24]. Sniffing sticks are a useful olfactory test (fig. 1).

There is no difference between patients with the tremor-dominant, rigid-akinetic or equivalence type; all of them present with hyposmia [24]. Thus, hyposmia is a common phenomenon which is present before motor symptoms occur [25]. Specificity is not $100 \%$, but this also applies to tremor, rigidity and bradykinesia. Even resting tremor can be a monosymptomatic feature and may never convert to PD. It may be helpful, however, for the categorization of a parkinsonian syndrome to take into consideration that whilst olfactory testing is generally abnormal in idiopathic PD, it is normal in PD due to parkin defects [26], in vascular PD [27] and in toxin-in-
Table 1. Non-motor features in PD

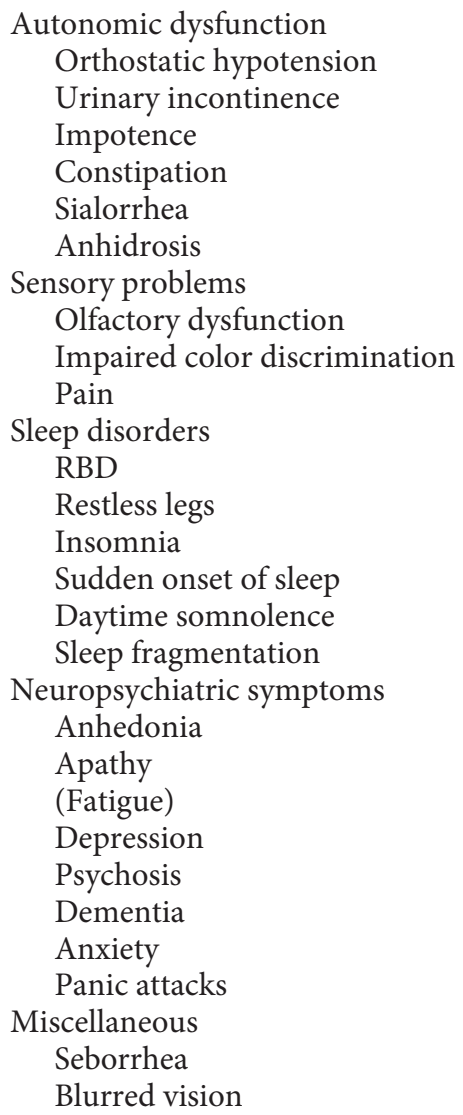

duced PD such as MPTP-induced PD [28]. We and others have shown that olfactory tests may be a useful tool to identify PD in the so-called premotor phase [29-32]. Our own group has shown that patients who lose their sense of smell in their mid-50s have an increased risk for the later development of PD. We found that amongst $30 \mathrm{pa}-$ tients with hyposmia or anosmia one patient presented with typical PD; with the additional use of transcranial parenchymal sonography, 11 patients were found to have hyperechogenicity of the midbrain, which was shown by Becker et al. [33] to be a classical sign of PD. Of these 11 patients with hyperechogenicity, 6 showed a reduced dopamine transporter activity on functional PET scanning [30]. Some years later, 2 of those patients had also developed motor features of PD [31]. Ponsen et al. [29] performed studies in an even larger cohort, and found that about $10 \%$ of patients who presented with hyposmia developed typical PD several years later. In conclusion, hyposmia is certainly a cardinal symptom of $\mathrm{PD}$, similar to the more classical ones described by Hughes et al. [2]. Un- 
Fig. 1. Sniffin' Stick set.

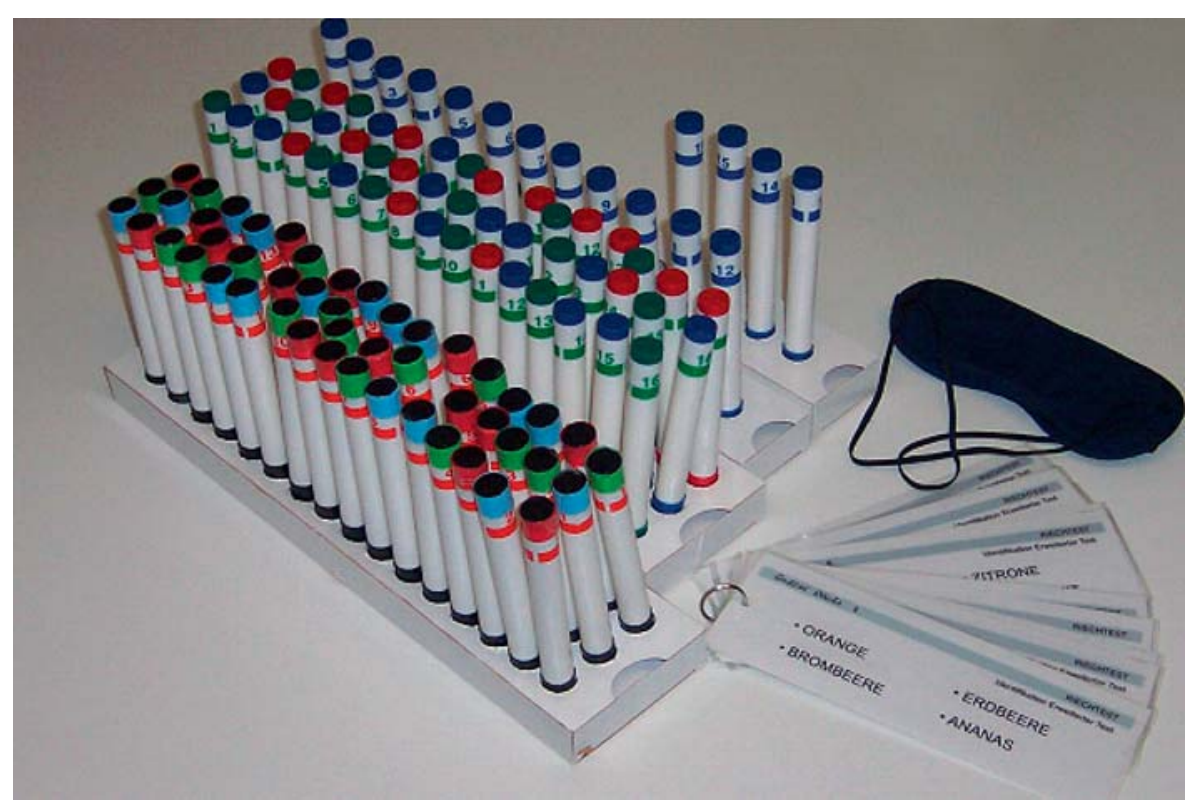

fortunately, hyposmia is not a good marker for cell degeneration because it changes in an unpredictable manner during the course of the disease [34].

Other early signs of PD are depression, constipation and REM sleep behavioral disorder (RBD). Iranzo et al. [35] assessed retrospectively 44 consecutive patients with a history of RBD of at least 2 years. They showed that $45 \%$ of patients with RBD have developed a neurological disorder at a mean follow-up of 5.1 years with emerging diagnoses of PD in 9 patients, dementia with Lewy bodies in 6 patients, cerebellar type multiple system atrophy in 1 patient and mild cognitive impairment in 4 patients. Thus, close follow-up of patients with idiopathic RBD may facilitate early diagnosis of neurodegenerative diseases. Stiasny-Kolster et al. [36] combined RBD with olfactory dysfunction as a possible indicator for PD and other $\alpha$-synucleinopathies. They could show that RBD patients have a profound impairment of olfaction and that may reflect stage 2 according to the Braak criteria for PD [19]. Besides urogenital, cardiovascular and thermoregulatory problems [37], patients suffer from gastrointestinal problems such as constipation. According to Braak's hypothesis, the first Lewy bodies occur in the Meissner and Auerbach plexuses, which would explain the loss or decrease in gut motility [22] which is the cause of the unpredictable absorption of anti-Parkinson medication. Constipation occurs in many patients with PD before the onset of motor symptoms [38]; the same is also true for RBD $[39,40]$. Finally, depression precedes motor symptoms in
$30 \%$ of patients with PD [41, 42]. Reijnders et al. [43] have described major depression, minor depression and dysthymia in 17, 22 and $13 \%$ of PD patients, respectively. In a survey of German PD patients, we found that only about a third of all PD patients did not present with psychiatric problems (depression, psychosis or dementia) when they were screened using rating scales [44]. This indicates that all patients with depression should undergo a careful neurological examination to check for PD symptoms. All of these symptoms are non-motor and not very sensitive and specific, and therefore an inexpensive screening test for PD would be desirable. Most recent reports [45] claim that melanine is elevated in peripheral blood in patients with $\mathrm{PD}$ and is an indicator of dopaminergic neuron breakdown. In this study, the authors compared PD patients with controls; 5 of the controls showed elevated melanine antibody binding and 3 of the 5 also had hyposmia. The authors report that after several years, 3 of the abnormal 5 'controls' had subtle signs of PD. Nonetheless, these preliminary reports have to be confirmed in more extensive studies before one can speculate on their value.

\section{Bedside Tests}

It is a common experience that levodopa and apomorphine tests are quite useful in establishing a diagnosis of PD. Most centers give $200 \mathrm{mg}$ levodopa/carbidopa in liquid form to the patient and expect to see an improvement 
in the Unified Parkinson Disease Rating Scale (UPDRS) of $30 \%$ or more. This definition, however, may be too restrictive in patients with a very mild form of PD. The apomorphine test is performed with $50 \mu \mathrm{g} / \mathrm{kg}$ body weight and should also result in an improvement in the UPDRS by $30 \%$ or more. It has to be stated, however, that the apomorphine challenge test remains controversial with low predictive values particularly in the Parkinson variant of multiple system atrophy and also progressive supranuclear palsy. Tremor does not necessarily have to improve in these tests, which once more underlines that parkinsonian resting tremor may not be due only to the dopaminergic deficit. Müller et al. [46] claim that the apomorphine test is less reliable than the levodopa test. Unfortunately, there are PD patients who show no response in either test. It is still unclear as to whether a levodopa test in young onset patients leads to priming. This putative priming phenomenon was seen in young onset patients who developed dyskinesias after a levodopa test had been performed years before, when they received, having been treated with dopamine agonists or other substances for months or years, again levodopa [47].

\section{Laboratory Tests}

Basic tests should include thyroid and liver function tests, and measurement of electrolytes and coeruloplasmine [48]. Copper and coeruloplasmine are relevant in PD cases with onset prior to 50 years.

The most recent studies [49] indicate that we may be at the advent of a lab test for PD. This has been suggested by a recent work on activated microglia in the basal ganglia [50]. It is of particular interest that the binding of anti-melanine antibodies was particularly high in patients in the early stages of the disease when presumably the majority of dopamine neurons die. As mentioned earlier, in the so-called control group some patients with elevated antibody titers and hyposmia later developed PD. Thus, there is hope that such a test may be a new and fascinating tool for the diagnosis of PD.

\section{Imaging}

CT or MRI scans are not useful in the diagnosis of idiopathic PD but rather to discriminate between atypical and symptomatic PD.

Dopamine transporter scans, dopamine receptor scans, meta-iodobenzyl-guanosine scans and glucose scans are helpful to reach a diagnosis in difficult cases where it is clinically impossible to differentiate between ET and PD or between idiopathic or atypical PD. The currently available diagnostic techniques such as dopamine transporter SPECT [51], F-Dopa PET [52] or even MIBG scintigraphy [53] certainly have a high sensitivity. The first two methods analyze dopamine function in the presynaptic neuron, and the MIBG scintigraphy demonstrates impairment of the postganglionic part of the sympathetic nervous system. The latter is abnormal in PD, but normal in atypical PD and in healthy persons. Patients with atypical PD have an impairment of the preganglionic part of the sympathetic nervous system. These nuclear medicine methods are useful not only for very early detection of PD but also for the differential diagnosis of mono-symptomatic tremor in PD versus ET. Most recently, Tang et al. [54] have shown that the use of fluorodeoxyglucose PET and an automated image-based classification procedure results in a high specificity in distinguishing between parkinsonian disorders.

\section{Genetic Tests}

In the future, we may have to consider PD as a genetic disease, although I doubt this. More than ten gene defects are known to be associated with a parkinsonian syndrome [55], which show similarities to the idiopathic Parkinson syndrome. It is extremely difficult to identify which patients should be tested, particularly because of the enormous costs of these analyses. In families with the LRRK2 deficiency [56], which has an autosomal-dominant trait, the problem will occur that some individuals will learn that they are positive for the causative point mutation decades before they may develop a PD syndrome. This problem resembles very much the problem of predictive genetic testing for Huntington's disease, and we will have to establish rules as to how to deal with such patients.

Maybe in the near future patients who have a relative with PD or who are younger than 45 could have a genetic microchip analysis performed.

\section{Additional Diagnostic Tests}

Many groups consider parenchymal sonography [35] as a supportive diagnostic tool because it shows mesencephalic hyperechogenicity contralateral to the clinically predominantly affected side. Unfortunately, the meth- 
od's sensitivity and specificity are far from 100\% [57], and frequently temporal bone thickness does not permit reliable testing. Quantitative tremor analysis may be used to assess tremor amplitudes and to unmask any tremor by using hand weights.

\section{Early Treatment in PD}

Recent recommendations suggest the initiation of PD treatment immediately after establishing the diagnosis. This view is supported by studies with rasagiline using a delayed start design $[58,59]$. It was shown in both studies that the early start of treatment is beneficial for the patients, probably due to disease modification by the MAO$\mathrm{B}$ inhibitor rasagiline. Lately, some retrospective analyses derived from trials with entacapone and rotigotine showed the same trend. A prospective study with the dopamine agonist pramipexole (PROUD study) has been published in poster form and was not positive, i.e. there was no difference in motor function and dopamine transporter imaging between patients with immediate and those with delayed initiation of therapy with pramipexole. These studies seem to indicate that patients should start treatment early. Furthermore, a study on quality of life issues [60] is rather interesting because it shows that patients who delayed therapy had a lower quality of life than those who started various treatments immediately. These studies allow the establishment of modern guidelines for the diagnosis of PD. It may become mandatory to establish the diagnosis of PD very early if a maximum disease-modifying or neuroprotective effect of the modern PD drugs is to be achieved.

In current clinical practice, clinical criteria for the diagnosis of PD will mostly remain to be motor symptoms, as discussed above. Additional non-motor symptoms which often develop later than motor symptoms will support a pre-existing diagnosis of PD. A useful tool for early diagnosis is the so-called levodopa test, where patients receive $200 \mathrm{mg}$ levodopa - this should improve their motor skills by at least $30 \%$. The clinical signs and the levodopa test will remain the corner-stones of PD diagnosis until further techniques emerge.

\section{References}

1 Alves G, Forsaa EB, Pedersen KF, et al: Epidemiology of Parkinson's disease. J Neurol 2008;255(suppl 5):18-32.

2 Hughes AJ, Daniel SE, Kilford L, Lees AJ: Accuracy of clinical diagnosis of idiopathic Parkinson's disease: a clinico-pathological study of 100 cases. J Neurol Neurosurg Psychiatry 1992;55:181-184.

3 Dickson DW, Braak H, Duda JE, et al: Neuropathological assessment of Parkinson's disease: refining the diagnostic criteria. Lancet Neurol 2009;8:1150-1157

4 Hoehn MM, Yahr MD: Parkinsonism. Onset, progression and mortality. Neurology 1967; 17:427-442.

5 Hallett M: Differential diagnosis of tremor; in Vinken PJ, Bruyn GW, Klawans HL (eds): Handbook of Clinical Neurology: Extrapyramidal Disorders, ed 49. New York, Elsevier Science, 1986, pp 583-595.

6 Rajput AH, Rozdilsky B, Ang L: Occurrence of resting tremor in Parkinson's disease. Neurology 1991;41:1298-1299.

7 Findley LJ, Gresty MA: Tremor. Br J Hosp Med 1981;26:16-32.

8 Clarimón J, Pagonabarraga J, Paisán-Ruiz C, et al: Tremor dominant parkinsonism: clinical description and LRRK2 mutation screening. Mov Disord 2008;15:518-523.
9 Antonini A, Vontobel P, Psylla M, et al: Complementary positron emission tomographic studies of the striatal dopaminergic system in Parkinson's disease. Arch Neurol 1995;52: 1183-1190.

10 Findley LJ, Gresty MA: Tremor and rhythmical involuntary movements in Parkinson's disease; in Findley LJ, Capildeo R (eds): Movement Disorders: Tremor. London, Macmillan, 1984, pp 295-304

11 Marsden CD: Slowness of movement in Parkinson's disease. Mov Disord 1989;4(suppl 1):S26-S37.

12 Agid Y: Parkinson's disease: pathophysiology. Lancet 1991;337:1321-1324.

13 Mendonca DA: Tasks of attention augment rigidity in mild Parkinson disease. Can J Neurol Sci 2008;35:501-505.

14 Weiner WJ, Nora LM, Glantz RH: Elderly inpatients: postural reflexes impairment. Neurology 1984;34:945-947.

15 Marttila RJ, Rinne UK: Disability and progression of Parkinson's disease. Acta Neurol Scand 1977;56:159-169.

16 Rajput AH, Rozdilsky B, Rajput A: Accuracy of clinical diagnosis in parkinsonism - a prospective study. Can J Neurol Sci 1991;18:275278.

17 Williams DR, Lees AJ: Progressive supranuclear palsy: clinicopathological concepts of diagnostic challenges. Lancet Neurol 2009;8: 270-279.
18 Shulman LM, Taback RL, Bean J, Weiner WJ: Comorbidity of the nonmotor symptoms of Parkinson's disease. Mov Disord 2001;16: 507-510.

19 Braak H, DelTredici K, Rub U, et al: Staging of brain pathology related to sporadic Parkinson's disease. Neurobiol Aging 2003;34: 197-211.

20 Jellinger K: Recent developments in the pathology of Parkinson's disease. J Neural Transm 2002;62(suppl):347-376.

21 Wakabayashi K, Takahashi H, Takeda S, Ohama E, Ikuta F: Parkinson's disease: the presence of Lewy bodies in Auerbach's and Meissner's plexuses. Acta Neuropathol 1988; 76:217-221.

22 Braak H, de Vos RA, Bohl J, Del Tredici K: Gastric alpha-synuclein immunoreactive inclusions in Meissner's and Auerbach's plexuses in cases staged for Parkinson's diseaserelated brain pathology. Neurosci Lett 2006; 20:67-72.

23 Müller A, Reichmann H, Livermore A, Hummel T: Olfactory function in idiopathic Parkinson's disease (IPD): results from cross-sectional studies in IPD patients and long-term follow-up of de novo IPD patients. J Neural Transm 2002;109:805-811. 
24 Doty RL, Deems DA, Stellar S: Olfactory dysfunction in parkinsonism: a general deficit unrelated to neurologic signs, disease stage, or disease duration. Neurology 1988;38: 1237-1244.

25 Haehner A, Boesveldt S, Berendse HW, Reichmann H, et al: Prevalence of smell loss in Parkinson's disease - a multicenter study. Parkinsonism Relat Disord 2009;15:490494.

26 Khan NL, Katzenschlager R, Watt H, et al: Olfaction differentiates parkin disease from early-onset parkinsonism and Parkinson disease. Neurology 2004;62:1224-1226.

27 Katzenschlager R, Zijlmans J, Evans A, Watt $\mathrm{H}$, Lees AJ: Olfactory function distinguishes vascular parkinsonism from Parkinson's disease. J Neurol Neurosrug Psychiatry 2004;75:1749-1752

28 Doty RL, Singh A, Tetrud J, Langston JW: Lack of major olfactory dysfunction in MPTP-induced parkinsonism. Ann Neurol 1992;32:97-100.

29 Ponsen MM, Stoffers B, Booji J, et al: Idiopathic hyposmia as a preclinical sign of Parkinson's disease. Ann Neurol 2004;56:173181.

30 Sommer U, Hummel T, Cormann K, et al: Detection of presymptomatic Parkinson's disease: combining smell tests, transcranial sonography, and SPECT. Mov Disord 2004; 19:1196-1202.

31 Haehner A, Hummel T, Hummel C, et al: Olfactory loss may be a first sign of idiopathic Parkinson's disease. Mov Disord 2007;22: 839-842.

32 Ross GW, Petrovitch H, Abbott RD, et al: Association of olfactory dysfunction with risk for future Parkinson's disease. Ann Neurol 2008;63:167-173.

33 Becker G, Seufert J, Bogdahn U, Reichmann $\mathrm{H}$, Reiners K: Degeneration of substantia nigra in chronic Parkinson's disease visualized by transcranial color-coded real-time sonography. Neurology 1995;45:182-184.

34 Herting B, Schulze S, Reichmann H, Haehner A, Hummel T: A longitudinal study of olfactory function in patients with idiopathic Parkinson's disease. J Neurol 2008;255: 367-370.

35 Iranzo A, Molinuevo JL, Santamaria J, et al: Rapid-eye-movement sleep behaviour disorder as an early marker for a neurodegenerative disorder: a descriptive study. Lancet Neurol 2006;5:572-577.
36 Stiasny-Kolster K, Doerr Y, Moeller CJ, et al: Combination of 'idiopathic' REM sleep behaviour disorder and olfactory dysfunction as possible indicator for alpha-synucleinopathy demonstrated by dopamine transporter FP-CIT-SPECT. Brain 2005;128:126-137.

37 Ziemssen T, Reichmann H: Non-motor dysfunction in Parkinson's disease. Parkinsonism Relat Disord 2007; 13:323-332.

38 Abbott RD, Petrovitch H, White LR, et al: Frequency of bowel movements and the future risk of Parkinson's disease. Neurology 2001;57:456-462.

39 Comella CL, Nardine TM, Diederich NJ, Stebbins GT: Sleep-related violence, injury, and REM sleep behaviour disorder in Parkinson's disease. Neurology 1998;51:526529.

40 Schenck CH, Brundlie SR, Mahowald WM: Delayed emergence of a parkinsonian disorder in $38 \%$ of 29 older men initially diagnosed with idiopathic rapid eye movement sleep behaviour disorder. Neurology 1996; 46:388-393.

41 Santamaria J, Tolosa E, Valles A: Parkinson's disease with depression; a possible subgroup of idiopathic parkinsonism. Neurology 1986;36:1130-1133.

42 Leentjens AF, van den Akker M, Metsemakers JF, Lousberg R, Verhey FR: Higher incidence of depression preceding the onset of Parkinson's disease: a register study. Mov Disord 2003;18:414-418.

43 Reijnders JS, Ehrt U, Weber WE, Aarsland D, Leentjens AF: A systemic review of prevalence studies of depression in Parkinson's disease. Mov Disord 2008;23:183-189.

44 Riedel O, Klotsche J, Spottke A, et al: Cognitive impairment in 873 patients with idiopathic Parkinson's disease. Results from the German Study on Epidemiology of Parkinson's Disease with Dementia (GEPAD). J Neurol 2008;255:255-264.

45 Double KL, Rowe DB, Carew-Jones FM, et al: Anti-melanin antibodies are increased in sera in Parkinson's disease. Exp Neurol 2009;217:297-301.

46 Müller T, Benz S, Boernke CH, et al: Worsened motor test performance following acute apomorphine injection in previously untreated patients with Parkinson's disease. J Neural Transm 2004;68(suppl):79-87.

47 Nadjar A, Gerfen CR, Bezard E: Priming for 1-dopa-induced dyskinesia in Parkinson's disease: a feature inherent to the treatment or the disease? Prog Neurobiol 2009;87:1-9.
48 Diener $\mathrm{CH}$, et al: Leitlinien für Diagnostik und Therapie in der Neurologie. Stuttgart, Thieme, 2008.

49 Double KL, Rowe DB, Carew-Jones FM, et al: Anti-melanin antibodies are increased in sera in Parkinson's disease. Exp Neurol 2009;217:297-301.

50 Gerhard A, Pavese N, Hotton G, et al: In vivo imaging of microglial activation with $[11 \mathrm{C}]^{\circledR}{ }_{-}$ PK11195 PET in idiopathic Parkinson's disease. Neurobiol Dis 2006;21:404-412.

51 Parkinson Study Group: Pramipexole vs. levodopa as initial treatment for Parkinson disease: a randomized controlled trial. JAMA 2000;284:1931-1938.

52 Whone AL, Bailey DL, Remy P, Pavese N, Brooks DJ: A technique for standardized central analysis of 6-(18)F-fluoro-L-DOPA PET data from a multicenter study. J Nucl Med 2004;45:1135-1145.

53 Braune S, Reinhardt M, Schnitzer R, Riedel A, Lücking $\mathrm{CH}$ : Cardiac uptake of [123] MIBG separates Parkinson's disease from multiple system atrophy. Neurology 1999;53: 1020-1025.

54 Tang CC, Poston KL, Eckert T, et al: Differential diagnosis of parkinsonism: a metabolic imaging study using pattern analysis. Lancet Neurol 2010;9:149-158.

55 Biskup S, Gerlach M, Kupsch A, Reichmann $\mathrm{H}$, et al: Genes associated with Parkinson syndrome. J Neurol 2008;255(suppl 5):8-17.

56 Hardy J, Lewis P, Revesz T, Lees A, PaisanRuiz C: The genetics of Parkinson's syndromes: a critical review. Curr Opin Genet Dev 2009; 19:254-265.

57 Vlaar AMM, de Nijs T, van Kroonenburgh $\mathrm{M}$, et al: The predictive value of transcranial duplex sonography for the clinical diagnosis in undiagnosed parkinsonian syndromes: comparison with SPECT scans. BMC Neurology 2008;8:42.

58 Parkinson Study Group: A controlled trial of rasagiline in early Parkinson disease: the TEMPO study. Arch Neurol 2002;59:19371943.

59 Olanow CW, Rascol O, Hauser R, et al: A double-blind, delayed-start trial of rasagiline in Parkinson's disease. N Engl J Med 2009;361:1268-1278.

60 Grosset D, Taurah L, Burn DJ, et al: A multicentre longitudinal observational study of changes in self reported health status in people with Parkinson's disease left untreated at diagnosis. J Neurol Neurosurg Psychiatry 2007;78:465-469. 